\title{
Exercise rehabilitation in the fourth industrial revolution
}

\author{
Yong-Seok Jee (iD http://orcid.org/0000-0001-6797-0843
}

The fourth industrial revolution was mentioned in the World Economic Forum in 2016. It has become a term representing the new industrial age based on information and communication technology. The third industrial revolution (information revolution) was characterized by the widespread use of computers and the Internet. The fourth industrial revolution, characterized by hyperconnectivity and superintelligence, is considered to be more advanced and widespread than the previous industrial revolution. Intelligent information technologies of the current industrial revolution include artificial intelligence, Internet of things, cloud computing, big data, and mobile convergence on existing industries and services. In particular, it combines all the products and services of the real world with new technologies in various fields such as 3-dimensional printing, robotics, biotechnology, and nanotechnology.

Exercise rehabilitation is generally prescribed to patients with various illnesses. Recently, exercise rehabilitation has been widely used for disorders that affect many parts of the body, such as neuromuscular dysfunction and cerebral dysfunction. Myelodysplastic syndrome refers to diseases with generalized muscular dystrophy, decreased muscle tone, muscular spasms, and severe pain in the muscles due to weakness of the upper or lower limbs which is related to hereditary, inflammatory, endocrine, and metabolic causes (Grisold and Vass, 2012).

Since muscular system diseases limit movement, a long period of resting time is needed, which makes regular exercise, going outdoors, and symptom management and treatment very difficult (Sackley et al., 2009). Although exercise rehabilitation with therapeutic purposes depends on the extent of the disease, it is general- ly desirable that several assistants participate in the program. Because of the many challenges nervous system patients face during exercise rehabilitation, intelligent robots are needed. Particularly, maintaining a patient's posture normally requires nanotechnology, which requires the handling of light materials, and biotechnology, that can help patients move easily. This allows the patient to find and adjust their proper posture to prevent and relieve muscle stiffness. The main goal of exercise rehabilitation is to allow people with neuromuscular disorders respond to a variety of abnormal stimuli, including minimizing or relaxing the alignment and symmetry of body posture.

In a study by Lee et al. (2011), muscle strength and range of motion (ROM) were increased after 5 weeks of robotic horse riding for $60 \mathrm{~min}$ in patients with myasthenia scoliosis of an 11-yearold thoracic lumbar spine compared with before starting treatment. Hammer et al. (2005) reported that exercise rehabilitation in patients with myopathy resulted in increased muscle tension, balance, and walking ability, but reduced spasticity and pain. In addition, some researchers claim that exercise rehabilitation is highly effective as a rehabilitation method for physical and mental deterioration caused by multiple sclerosis (Flachenecker, 2012).

Cerebral disease is one of the major causes of death worldwide, and it is one of the diseases that is increasing even in developed environments. Brain disease is a terrible illness that afflicts not only the patient, but also the people around him or her because it accompanies physical and mental disorders. Brain diseases are divided into various types, but they are mainly caused by stroke due to cerebrovascular disorder and dementia caused by genetic changes, cerebral palsy occurring in children and infants, and traumatic 
brain injury due to traffic accidents.

In general, brain diseases causes secondary problems accompanied by paralysis or dysfunction of the body and gait difficulties due to primary brain lesions. Therefore, it is necessary to reverse muscle atrophy caused by paralysis, improve the ROM, and overcome walking disorders. Among the subjects participating in the exercise rehabilitation in Korea and overseas, cerebral palsy is the most single common disease. In the case of cerebral palsy, children who grow up in the musculoskeletal system are more likely to be treated and have a shorter duration of treatment than adults.

There have been many studies on exercise rehabilitation for cerebral palsy in Korea and elsewhere. A summary of some of these studies suggests an improvement in major motor function, an increase in walking speed and stride length, and a reduction in pelvic joint stenosis due to paralysis. Most exercise rehabilitation professionals can reduce hip pain, stiffness, and protrusion of the hip joints in patients with cerebral palsy through a variety of exercise rehabilitation equipment. In addition, the effects of exercise rehabilitation on monoplegia, hemiplegia, and bilateral spastic cerebral palsy are very beneficial.

In the rapidly developing modern society, the concept of disability is regarded as a universal phenomenon that can happen to anybody. Therefore, it is urgent to combine exercise rehabilitation, which is performed with the purpose of treating people with disabilities, and various programs derived from the fourth industrial revolution. The various values obtained through this can offer a new paradigm for improving the quality of life and longevity of patients.

\section{CONFLICT OF INTEREST}

No potential conflict of interest relevant to this article was reported.

\section{REFERENCES}

Flachenecker P. Autoimmune diseases and rehabilitation. Autoimmun Rev 2012;11:219-225.

Grisold W, Vass A. Neuromuscular complications. Handb Clin Neurol 2012;105:781-803.

Hammer A, Nilsagård Y, Forsberg A, Pepa H, Skargren E, Oberg B. Evaluation of therapeutic riding (Sweden)/hippotherapy (United States). A single-subject experimental design study replicated in eleven patients with multiple sclerosis. Physiother Theory Pract 2005;21:51-77.

Lee DR, Lee NG, Cha HJ, Yun Sung O, You SJ, Oh JH, Bang HS. The effect of robo-horseback riding therapy on spinal alignment and associated muscle size in MRI for a child with neuromuscular scoliosis: an experimenter-blind study. NeuroRehabilitation 2011;29:23-27.

Sackley C, Disler PB, Turner-Stokes L, Wade DT, Brittle N, Hoppitt T. Rehabilitation interventions for foot drop in neuromuscular disease. Cochrane Database Syst Rev 2009;(3):CD003908.

Research Institute of Sports and Industry Science, Hanseo University, 46 Hanseo 1-ro, Haemi-myeon, Seosan 31962, Korea E-mail: jeeys@hanseo.ac.kr 